\title{
Simulation and Experimental Study of Closed Crack Detection by Ultrasonic Nonlinearity Under Electromagnetic Loading
}

\author{
Chuang Zhang ${ }^{\mathrm{a}, \mathrm{b}, 1}$, Longlong He ${ }^{\mathrm{a}, \mathrm{b}}$, Suzhen Liu ${ }^{\mathrm{a}, \mathrm{b}}$, and Qingxin Yang ${ }^{\mathrm{a}}$ \\ a State Key Laboratory of Reliability and Intelligence of Electrical Equipment, Hebei \\ University of Technology, Tianjin, 300130 China \\ ${ }^{\mathrm{b}}$ Key Laboratory of Electromagnetic Field and Electrical Apparatus Reliability of \\ Hebei Province, Hebei University of Technology, Tianjin, 300387 China
}

\begin{abstract}
The reliability of micro-damage detection of metal materials plays a crucial role in the safe and reliable operation of large equipment. In recent years, nonlinear ultrasonic nondestructive testing technology has achieved good development in closed cracks detection, but the problem of nonlinear ultrasonic detection of closed cracks is weak response signal and vulnerable to external interference. This paper realizes the modulation of the ultrasonic wave by electromagnetic loading at the closed cracks, which can effectively enrich the frequency components of nonlinear ultrasonic and magnify the amplitude. This lays a foundation for further research on nonlinear ultrasonic detection of closed cracks under electromagnetic loading.
\end{abstract}

Keywords. Closed crack, electromagnetic loading, nondestructive testing, nonlinear ultrasound

\section{Introduction}

Metal structure is widely used in various major infrastructures, such as ship shell, train body, aircraft door, tank shell, etc. In service period, these metal structures are subject to the combined action of surroundings, temperature and cyclic load, and are prone to generate plastic damage and closed cracks. Statistical data shows that closed crack is one of the main forms of early fatigue damage in metal structures [1].

The accumulation of early fatigue damage takes up most of the time during the service of the structure, so the detection and evaluation of early fatigue damage is of great importance. Therefore, how to achieve the effective detection of closed cracks is also the focus of research in the field of non-destructive flaw detection [2]. There have been a lot of studies on the detection of macroscopic cracks and defects of metal plates by linear ultrasound [3]-[4]. However, it is difficult to detect the closed cracks by using linear ultrasound because the closed cracks are usually in contact and closed state. In recent years, nonlinear ultrasonic methods have been widely used to detect closed cracks, of which the sensitivity to closed cracks is higher. HJ Lim [5] used nonlinear ultrasonic modulation technology to detect metal plates containing fatigue cracks under different temperature and load conditions, indicating that the nonlinear ultrasonic modulation

${ }^{1}$ Corresponding Author Chuang Zhang, Email czhang@hebut.edu.cn. 
technology can accurately detect fatigue damage of metal structures under complex environment. H Tohmyoh [6] applied the surface SH wave to detect the fatigue crack on the surface of low carbon steel, and made a quantitative evaluation on the length and depth of the crack. Ikeuchi [7] proposed a method of fundamental wave amplitude difference based on the fundamental wave threshold caused by close cracks contact vibration, which can selectively image closed cracks and eliminate the influence of other linear scatters.

The main problem of closed cracks detection by nonlinear ultrasound is weak response signal. The application of electromagnetic loading technology to the nonlinear ultrasonic detection of closed cracks can not only avoid the additional damage that may be introduced by the mechanical loading to the tested components, but also effectively improve the reliability of the ultrasonic nonlinear detection of closed cracks [8]. In this paper, the closed cracks in metal plates are excited into dynamic fluctuation state through electromagnetic loading to improve the response signal of nonlinear ultrasound. Electromagnetic loading device is compact and portable; therefore, it is easier to operate than mechanical loading. The nonlinear modulation effect of closed cracks in metal plates under electromagnetic loading is verified by simulation and experiments. The influence of different loading frequencies on nonlinear detection of closed cracks is also studied. This provides a basis for the ultrasonic non-linear detection of closed cracks.

The paper is organized as follows. In Section 2, simulation of ultrasonic modulation under electromagnetic loading is discussed. In Section 3, the specimen preparation and experimental setup are described, and the verification experiment of VAM based on electromagnetic loading is elaborated, and Section 4 summarizes the conclusions of the study.

\section{Simulation}

\subsection{Ultrasonic modulating simulation under electromagnetic loading}

Fig. 1 is a schematic diagram of the ultrasonic modulating simulation model of an aluminum plate with a closed crack under electromagnetic loading. The COMSOL finite element simulation software is used for finite element analysis. The simulation model includes ultrasonic excitation devices (excitation coils and U-shaped magnets), electromagnetic loading devices (loading coils and rectangular permanent magnets), and an aluminum plate containing a closed crack.

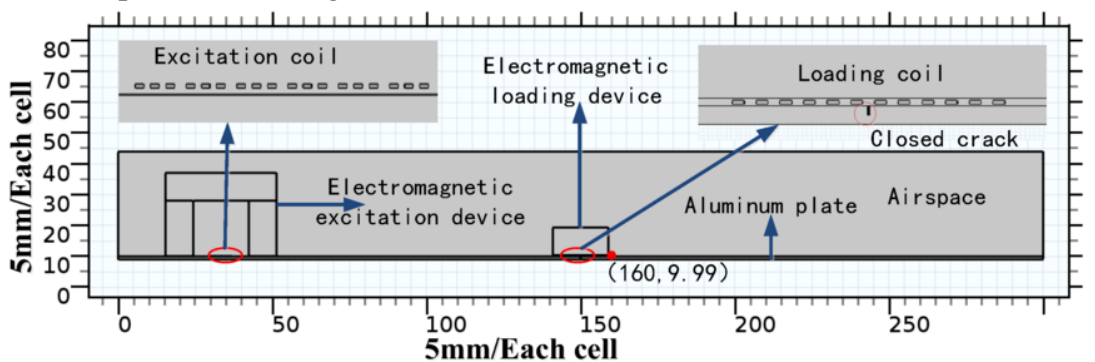

Fig. 1. Ultrasonic modulating model of aluminum plate with a closed crack under electromagnetic loading.

The exciting coil adopts a zigzag-coil, and the loading coil adopts a spiral coil. The closed crack is located at $(150,9.5)$ of the aluminum plate, of which the depth is $0.5 \mathrm{~mm}$. 
The permanent magnet of the electromagnetic loading devices generates a vertical downward magnetic field with a residual magnetic flux density of $1 \mathrm{~T}$. The line spacing of the loading coil is set at $0.5 \mathrm{~mm}$ and the number of turns is 12 . A sinusoidal current with a peak-to-peak amplitude of $10 \mathrm{~A}$ is loaded into the loading coil, of which the frequency is $200 \mathrm{kHz}$. The magnet of the electromagnetic ultrasonic transducer is a Ushaped magnet, and the residual magnetic flux density is set at $1 \mathrm{~T}$. The excitation coil adopts a three-split wire with a turn of 6 , of which the distance between the bundles is set at $1.17 \mathrm{~mm}$ and the distance between splitting wires is $0.15 \mathrm{~mm}$. The excitation current is a Gaussian window modulated sine pulse excitation, which has a frequency of $1 \mathrm{MHz}$, a peak-to-peak amplitude of $10 \mathrm{~A}$, and a circulation of 10 . The node B (160, 9.99) on the aluminum plate is used as the signal acquisition point to simulate the ultrasonic modulating effect and analyze the influence of electromagnetic loading parameters on the electromagnetic ultrasonic wave during the propagation in the aluminum plate.

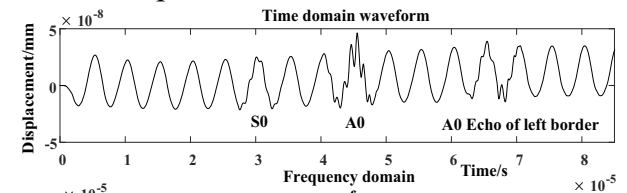

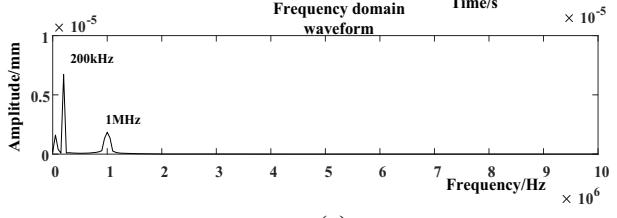

(a)
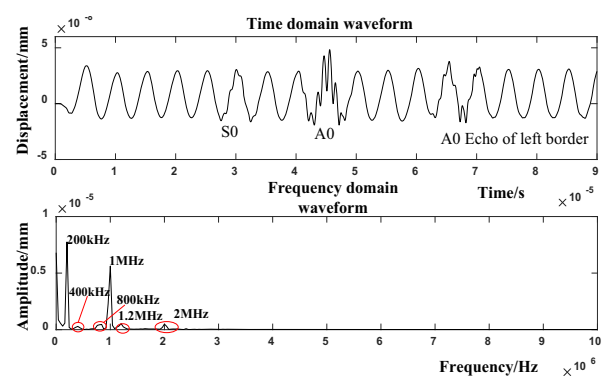

(b)

Fig. 2. Time-frequency diagram of intact (a) and defective (b) aluminum plate.

Fig. 2 shows the ultrasonic time-domain waveforms and spectrograms acquired at point $\mathrm{B}$ with or without closed cracks and with or without electromagnetic loading. As shown in Fig 2(a), when electromagnetic loading is performed in an intact metal plate, the time domain waveform is a superposition of an electromagnetic loading signal and an ultrasonic signal, and the frequency domain signal contains only a $200 \mathrm{kHz}$ loading frequency and $1 \mathrm{MHz}$ ultrasonic frequency. Fig. 2(b) shows the time-domain waveform and frequency spectrum of the $1 \mathrm{MHz}$ ultrasonic signal propagating in an aluminum plate with a closed crack under $200 \mathrm{kHz}$ electromagnetic loading. It can be clearly seen that the time domain waveform of ultrasonic signal and loading signal has superimposed. Due to the crack fluctuation and electromagnetic loading, the time-domain signal of the received signal is distorted. In addition to the $200 \mathrm{kHz}$ and $1 \mathrm{MHz}$ fundamental signals in the spectrum, there are also the second harmonic components of $400 \mathrm{kHz}$ and $2 \mathrm{MHz}$ and the sum and difference components of $1.2 \mathrm{MHz}$ and $800 \mathrm{kHz}$.

The analysis above shows that the crack interface undergoes microscopical openclose fluctuation under electromagnetic loading, and the fluctuation will modulate the ultrasonic waves propagating in the aluminum plate. When the ultrasonic wave passes through the closed crack region, it will be strongly modulated. The time domain waveform is distorted, and the modulating frequency components appear in the frequency spectrum. A typical feature is that the sum and difference frequency components appear in the frequency spectrum. By comparing the frequency domain diagrams of the ultrasonic signal with or without electromagnetic loading, it can be seen that the nonlinear components generated by electromagnetic loading are more abundant and have higher amplitude. Therefore, it can be concluded that the detection of closed 
cracks can be achieved by detecting the existence of sum frequency and difference frequency components of the ultrasonic response signal of the aluminum plate under electromagnetic loading.

\subsection{Influence of electromagnetic loading frequencies on ultrasonic modulation}

Changes in electromagnetic loading parameters will affect the eddy current in the aluminum plate. The Lorentz force is determined by the size and distribution of the eddy current. This changes the fluctuating state of the closed crack, which will then affect the ultrasonic waves propagating in it. The following analyze will focus on the influence of electromagnetic loading parameters on nonlinear ultrasonic modulation.
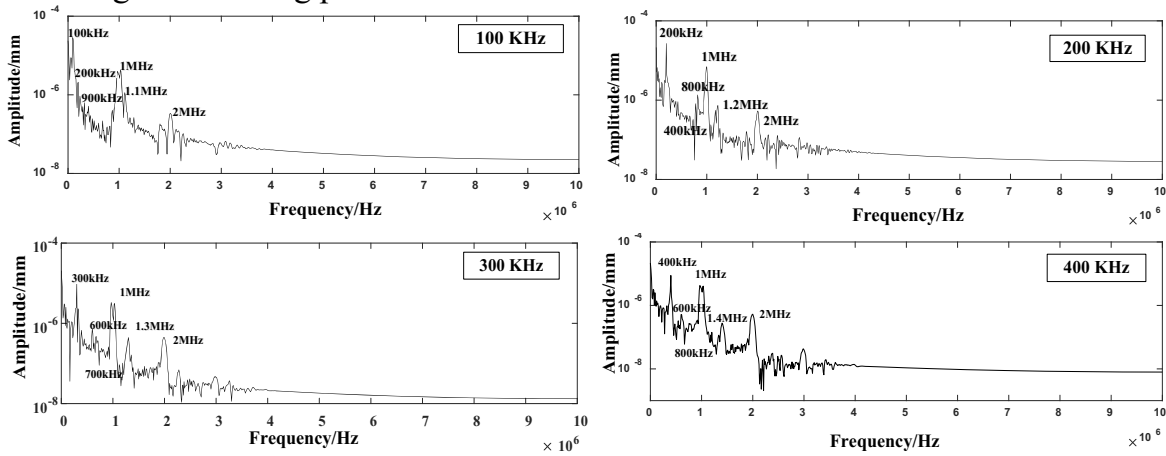

Figure 3. Frequency diagram at different loading frequencies.

The frequency of electromagnetic ultrasonic excitation is $1 \mathrm{MHz}$. The peak-to-peak amplitude of pulse current is $10 \mathrm{~A}$ and the excitation period is 10 cycles. The electromagnetic loading current is kept unchanged at $10 \mathrm{~A}$, and the loading frequency changes. $100 \mathrm{kHz}, 200 \mathrm{kHz}, 300 \mathrm{kHz}$, and $400 \mathrm{k} \mathrm{Hz}$ are used to simulate and analyze the characteristics of ultrasonic response at different loading frequencies. The results are shown in Fig. 3. When the electromagnetic loading frequency is $100 \mathrm{kHz}$, the loading frequency is too small compared with the exciting ultrasonic frequency, which leads to the overlap of $1.1 \mathrm{MHz}$ sum frequency component, $900 \mathrm{kHz}$ difference frequency component and $1 \mathrm{MHz}$ ultrasonic frequency. When the loading frequency is $300 \mathrm{kHz}$, the second harmonic component of $600 \mathrm{kHz}$ is generated due to the closed crack, which cannot be easily distinguished from the difference frequency component of $700 \mathrm{kHz}$. When the frequency is $200 \mathrm{kHz}$ or $400 \mathrm{kHz}$, the side-lobe and harmonic components can be easily distinguished. Thus, they can be used as the loading frequency.

\section{Experiments and analysis}

This section will verify the ultrasonic modulation of closed cracks in aluminum plates under electromagnetic loading through experiments. The tested specimens are an intact aluminum plate and an aluminum plate with a closed crack. As is shown in Fig. 4, the dimension of both is $300 \mathrm{~mm} \times 60 \mathrm{~mm} \times 1 \mathrm{~mm}$, and the crack is located at $150 \mathrm{~mm}$ to the left of the defective plate. The defective piece includes two kinds of defects, namely cutting defects and closed cracks, which are shown in Fig. 4. The manufacturing process of the defective piece is as follows: First, a wire cutting is used to make the cutting defect in the middle of the plate, of which the length is $5 \mathrm{~mm}$ and the width is $0.5 \mathrm{~mm}$; then it is 
fixed in a fatigue tester for cyclic loading. The frequency of cyclic loading is $20 \mathrm{~Hz}$ and the force is $5 \mathrm{kN}$. A closed crack with a length of $15 \mathrm{~mm}$ (as shown in Fig. 4) appears in the aluminum plate after loading up to 30,000 times. The experiment is performed on the intact aluminum plate and the defective aluminum plate. The connection of the experimental equipment is shown in Fig. 4. The electromagnetic loading device is placed directly above the closed crack. The vertical magnetic field produced by the permanent magnet of the loading device is approximately $0.3 \mathrm{~T}$. The loading coil adopts a spiral coil. The high-power signal source provides continuous sinusoidal excitation for the electromagnetic loading coil, of which the loading frequency is $200 \mathrm{kHz}$ and the current is $0.75 \mathrm{~A}$.
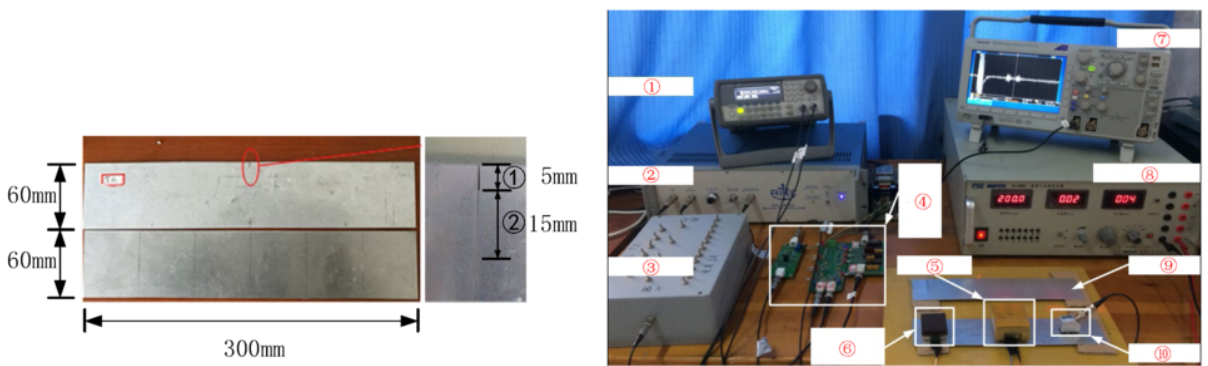

Fig. 4. Tested specimen and experimental equipment wiring diagram.

The electromagnetic ultrasonic exciting device is set $30 \mathrm{~mm}$ away from the left side of the aluminum plate. The magnetic field intensity provided by the permanent magnet of the ultrasonic exciting device is $0.4 \mathrm{~T}$. The exciting coil adopts a zigzag-coil. The tone-burst signal generated by the signal generator is amplified by the pulse amplifier and then output to the ultrasonic exciting coil. The impedance matching device matches the internal impedance of the exciting coil and the exciting generator so that the output of the signal reaches the maximum power. The peak-to-peak amplitude of the exciting signal is set at $350 \mathrm{mV}$, the frequency is $1 \mathrm{MHz}$, and the number of exciting cycles is 10 . The response signal is received using a piezo probe with a central frequency of $2.5 \mathrm{MHz}$. The probe is put at $200 \mathrm{~mm}$ away from the left of the tested aluminum plate. The position of the electromagnetic ultrasonic exciting device and the piezoelectric probe is approximately in line with the top of the closed crack. The received signal is output to the oscilloscope after a preamplifier, a wideband amplifier, and a low-pass filtering one after another.

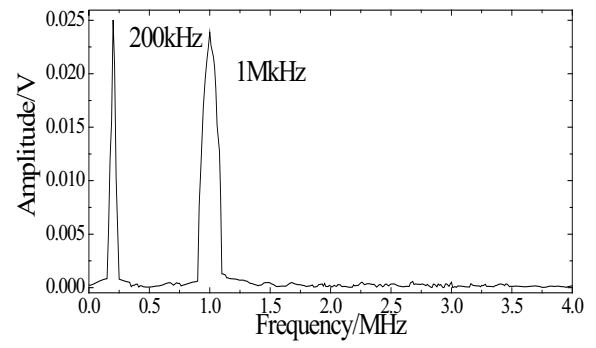

(a)

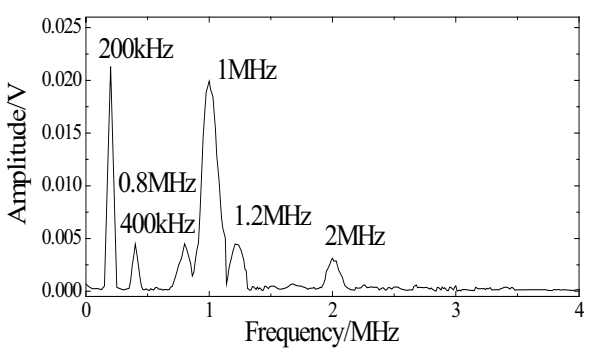

(b)

Fig. 5. Frequency domain diagram of the intact and defective aluminum plate.

By analyzing the frequency domain characteristics of the ultrasonic signal of the two specimens shown in Fig. 5, it can be seen that there are only $1 \mathrm{MHz}$ and $200 \mathrm{kHz}$ fundamental frequency components in the spectrum of the signal collected from the 
intact aluminum plate, and no significant harmonic components is collected. The spectrum of the signal collected from the defective aluminum plate includes not only the fundamental frequency components but also the second harmonics of $400 \mathrm{kHz}$ and 2 $\mathrm{MHz}$ and the sidelobe components of $800 \mathrm{kHz}$ and $1.2 \mathrm{MHz}$. The Lorentz force generated by electromagnetic loading causes the closed crack to open and close microscopically. The fluctuation of the closed crack will modulate the electromagnetic ultrasonic signal propagating through the crack, which produces a nonlinear modulating effect on the ultrasonic response.

\section{Conclusion}

To solve the problem of nondestructive detection of the closed cracks, this paper proposes to excite the aluminum plate with closed cracks into dynamic fluctuation by electromagnetic loading. Simulations and experiments show that: (1) Compared with traditional nonlinear ultrasonic testing, ultrasonic modulation under electromagnetic loading can excite richer and higher-amplitude nonlinear components, which increases the reliability of ultrasonic nonlinear detection of closed cracks. (2) The electromagnetic loading frequency should be less than half of the ultrasonic signal frequency, but higher than one tenth of it. This lays a foundation for further research on nonlinear ultrasonic detection of closed cracks under electromagnetic loading. Future work should focus on the elimination of nonlinear effects of equipment, the extraction of modulating signals, and the further optimization of loading parameters. These are very useful for the practical application of the electromagnetic loading ultrasonic modulating method to detect early fatigue damage of metal plates.

\section{References}

[1] Staszewski W J, Boller C, Tomlinson G. Health monitoring of aerospace structures: smart sensor technologies and signal processing. Chichester: John Wiley \& Sons, 2004.

[2] D. Broda, W. J. Staszewski, A. Martowicz et al., "Modelling of nonlinear crack-wave interactions for damage detection based on ultrasound-A review," J Sound Vib, vol. 333, no. 4, pp. 1097-1118, Feb. 2014.

[3] A. Mirala, R. S. Shirazi, "Detection of surface cracks in metals using time-domain microwave nondestructive testing technique," IET Microwaves Antennas Propag, vol. 11, no. 4, pp. 564-569, Mar. 2017.

[4] X. Xu, L. Zhuang et al., "An Ultrasonic Guided Wave Mode Excitation Method in Rails,” IEEE Access, vol. 6, pp. 60414-60428, Oct. 2018.

[5] H. J. Lim, H. Sohn, M. P. Desimio et al., "Reference-free fatigue crack detection using nonlinear ultrasonic modulation under various temperature and loading conditions," Mech Syst Signal Process, vol. 45, no. 2, pp. 468-478, Apr. 2014.

[6] H. Tohmyoh, Y. Ochi, T. Matsumura, "Study on detection and quantitative evaluation of fatigue cracks using surface SH waves (NDE2)," Nihon Kikai Gakkai Ronbunshu, A, vol. 67, no. 661, pp. 1508-1513, Aug. 2017.

[7] M. Ikeuchi, K. Jinno, Y. Ohara et al., "Improvement of closed crack selectivity in nonlinear ultrasonic imaging using fundamental wave amplitude difference," J. Appl. Phys, vol. 52, no. 7 PART 2, pp. 10441055, July. 2013.

[8] C. Zhang, W. Pang, S. Liu et al., "Dynamic characteristics analysis of closed cracks in aluminum plate under electromagnetic loading," Zhongguo Dianji Gongcheng Xuebao (Proceedings of The Chinese Society for Electrical Engineering), vol. 38, no. 7, pp. 2171-2178, Apr. 2018. (In Chinese). 